\title{
Real-Time Microfluidic Blood-Counting System for PET and SPECT Preclinical Pharmacokinetic Studies
}

\author{
Laurence Convert ${ }^{1-3}$, Réjean Lebel ${ }^{1}$, Suzanne Gascon ${ }^{1}$, Réjean Fontaine ${ }^{3}$, Jean-François Pratte ${ }^{3}$, Paul Charette ${ }^{2,3}$, \\ Vincent Aimez ${ }^{2,3}$, and Roger Lecomte ${ }^{1}$ \\ ${ }^{I}$ Sherbrooke Molecular Imaging Centre of CRCHUS and Department of Nuclear Medicine and Radiobiology, Université de \\ Sherbrooke, Sherbrooke, Quebec, Canada; ${ }^{2}$ Laboratoire Nanotechnologies Nanosystèmes (LN2)-CNRS UMI-3463, Université \\ de Sherbrooke, Sherbrooke, Quebec, Canada; and ${ }^{3}$ Institut Interdisciplinaire d'Innovation Technologique (3IT), Université de \\ Sherbrooke, Sherbrooke, Quebec, Canada
}

Small-animal nuclear imaging modalities have become essential tools in the development process of new drugs, diagnostic procedures, and therapies. Quantification of metabolic or physiologic parameters is based on pharmacokinetic modeling of radiotracer biodistribution, which requires the blood input function in addition to tissue images. Such measurements are challenging in small animals because of their small blood volume. In this work, we propose a microfluidic counting system to monitor rodent blood radioactivity in real time, with high efficiency and small detection volume $(\sim 1 \mu \mathrm{L})$. Methods: A microfluidic channel is built directly above unpackaged $p$-i-n photodiodes to detect $\beta$-particles with maximum efficiency. The device is embedded in a compact system comprising dedicated electronics, shielding, and pumping unit controlled by custom firmware to enable measurements next to small-animal scanners. Data corrections required to use the input function in pharmacokinetic models were established using calibrated solutions of the most common PET and SPECT radiotracers. Sensitivity, dead time, propagation delay, dispersion, background sensitivity, and the effect of sample temperature were characterized. The system was tested for pharmacokinetic studies in mice by quantifying myocardial perfusion and oxygen consumption with ${ }^{11} \mathrm{C}$-acetate (PET) and by measuring the arterial input function using $99 \mathrm{mTCO}_{4}{ }^{-}$(SPECT). Results: Sensitivity for PET isotopes reached $20 \%-47 \%$, a 2 - to 10 -fold improvement relative to conventional catheter-based geometries. Furthermore, the system detected $99 \mathrm{mTc}$-based SPECT tracers with an efficiency of $4 \%$, an outcome not possible through a catheter. Correction for dead time was found to be unnecessary for small-animal experiments, whereas propagation delay and dispersion within the microfluidic channel were accurately corrected. Background activity and sample temperature were shown to have no influence on measurements. Finally, the system was successfully used in animal studies. Conclusion: A fully operational microfluidic blood-counting system for preclinical pharmacokinetic studies was developed. Microfluidics enabled reliable and high-efficiency measurement of the blood concentration of most common PET and SPECT radiotracers with high temporal resolution in small blood volume.

Received Jun. 26, 2015; revision accepted Mar. 29, 2016.

For correspondence or reprints contact: Roger Lecomte, Department of Nuclear Medicine and Radiobiology, Université de Sherbrooke, 3001, 12th Ave. N., CRCHUS \#1977, Sherbrooke, Quebec, Canada J1H5N4. E-mail: Roger.Lecomte@USherbrooke.ca

Published online May 5, 2016.

COPYRIGHT (c) 2016 by the Society of Nuclear Medicine and Molecular Imaging, Inc.
Key Words: pharmacokinetic studies; microfluidic; arterial input function; small animal PET; small animal SPECT

J Nucl Med 2016; 57:1460-1466

DOI: $10.2967 /$ jnumed.115.162768

$\mathbf{R}$ adionuclide-based molecular imaging using PET and SPECT is a leading diagnostic tool in oncology, cardiology, and neurology (1). In research applications, small-animal models are needed to facilitate the development of new drugs, radiotracers, and therapies that can eventually be translated to humans (2). Quantification of metabolic or physiologic disorders using radiotracer pharmacokinetic models requires dynamic blood analysis in addition to tissue imaging data (3). Whole-blood radioactivity as a function of time, the so-called arterial input function (AIF), can be extracted from PET images $(4,5)$, using an intravascular $\beta$-microprobe $(6,7)$ or an external blood counter connected to an artery via a catheter $(8-10)$. For accurate quantification, the measured whole-blood AIF must be corrected for known plasmatic and metabolite fractions. For new radiotracers, such parameters are not yet established, and blood samples have to be drawn and analyzed. However, repetitive manual blood sampling is difficult and time-consuming and can affect the animal homeostasis, especially in mice $(\sim 1.4 \mathrm{~mL}$ total blood volume).

Lab-on-a-chip devices are miniature analysis platforms based on microelectronic fabrication principles that integrate one or several laboratory functions (11). They incorporate micron-scale fluidic channels that can perform several operations such as plasma separation, radiation detection, and chemical analysis. Thus, microfluidics technology is an attractive solution to provide full characterization of new radiotracers in small-animal studies. In this work, we present one building block of such an integrated microfluidic system for real-time blood radioactivity monitoring.

PET radiotracers emit positrons $\left(\beta^{+}\right)$, with a maximum energy $E_{\beta \max }$ ranging typically from 0.6 to $1.9 \mathrm{MeV}$, that rapidly annihilate, producing two 511-keV annihilation photons. Most SPECT radiotracers emit $\beta^{-}$and $\gamma$-rays $(60-250 \mathrm{keV})$ typically accompanied by Auger or conversion electrons (CEs, < $150 \mathrm{keV}$ ). Thus both PET and SPECT radiotracer concentration in blood can be monitored either by charged particle ( $\beta$, Auger, or CE) or by photon ( $\gamma$ - or annihilation) detection. High-energy photon detection requires high-density crystals, $1-2 \mathrm{~cm}$ thick, that are not appropriate 
for microsystem integration. Charged-particle detection is therefore preferred because it can be performed in a silicon wafer, the traditional substrate for microfluidic chips (12).

Charged-particle detection for blood radioactivity monitoring in pharmacokinetic studies faces several challenges. First, charged particles are stopped within a few micrometers to a millimeter in matter depending on their energy; $\mathrm{CE}$, Auger, and a significant part of the polyenergetic $\beta$-particles are severely attenuated at the interface between sample and detector. Second, radiotracer concentration in rodent blood is typically less than $100 \mathrm{~Bq} / \mu \mathrm{L}$ after tissue uptake. Third, in mice only a few hundred microliters at most are available for analysis over 10-45 min, hence detection volume must be kept as small as possible, around $1 \mu \mathrm{L}$, at any given time during measurement. Last, temporal resolution better than $4 \mathrm{~s}$ has been suggested as a prerequisite for accurate estimation of pharmacokinetic parameters (13). The detector must be able to measure a few $10 \mathrm{~s}$ events/s at a minimum to procure a statistically significant signal. Hence, detection efficiency must be maximized and the interface thickness between sample and detector minimized.

Examples of microfluidic systems for related applications were previously reported. Two microfluidic chips were developed for microblood sampling in PET imaging $(14,15)$, but no radiation detector was integrated. Samples had to be measured off-line in a wellcounter, which can be limiting for short-half-life radioisotopes, that is, ${ }^{15} \mathrm{O}(2 \mathrm{~min}),{ }^{13} \mathrm{~N}$ (10 min), or ${ }^{11} \mathrm{C}$ (20 min). Radiation detectors were used with microfluidic chips for DNA detection (16), protein adsorption (17), radiosynthesis monitoring (18), cell imaging (19), and protein crystallography (20), but with off-chip detectors. An early integrated chip design dedicated to charged-particle detection was proposed in the late 1990s for DNA detection (21), but the authors opted for fluorescence detection in the actual integration (22). More recently, a polydimethylsiloxane microfluidic chip was coupled to a position-sensitive avalanche-photodiode detector for cell imaging (23), but the interface thickness between sample and detector limited the sensitivity for PET tracers and did not allow the detection of lowenergy CE such as the ones emitted by ${ }^{99 \mathrm{~m}} \mathrm{Tc}$.

In previous work, our group built a microfluidic channel directly above an unpackaged $p-i-n$ silicon photodiode array, eliminating all unnecessary interfaces between blood and the photodiode detection layer to overcome these limitations $(24,25)$. We also proposed a method to improve the blood compatibility of the microchannel polymer building material (26). The microfluidic geometry was optimized, the fabrication process detailed, and a preliminary characterization of the microfluidic chip was performed (27). In the present work, we describe a prototype blood-counting system comprising the microfluidic chip together with dedicated electronics, software, and pumping unit. Data corrections required to use the measured AIF in pharmacokinetic models are described and fully characterized for the most common PET and SPECT radiotracers. Finally, the system was used for the measurement of myocardial perfusion and oxygen consumption in mice using the PET tracer ${ }^{11} \mathrm{C}$-acetate and to determine the AIF of the SPECT tracer ${ }^{99} \mathrm{~m} \mathrm{TcO}_{4}{ }^{-}$in mice.

\section{MATERIALS AND METHODS}

\section{System Overview}

The proposed microfluidic blood-counting system is based on direct $\beta$ - or electron detection within a microchannel built above unpackaged $p-i-n$ photodiodes. It consists of two separate hardware modules, a detection unit enclosed in a compact radiation shield linked to a peristaltic pump, and a control unit (Fig. 1). The supplemental materials

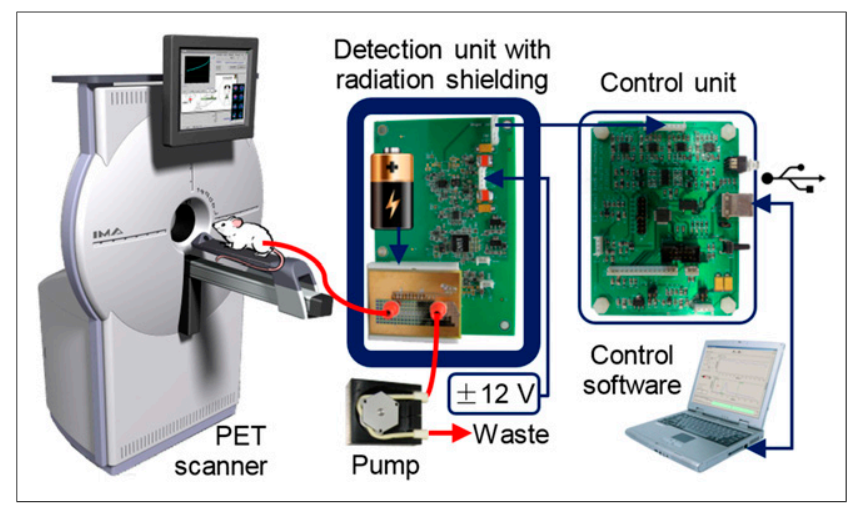

FIGURE 1. Schematic view of microfluidic blood-counting system.

provide a detailed description (supplemental materials are available at http://jnm.snmjournals.org).

\section{Microfluidic Detector}

The microfluidic chips were built with a rectangular microchannel forming a quasi-two-dimesional source and, therefore, achieving quasiperfect geometric detection efficiency (Fig. 2A). Furthermore, the microfluidic chips were designed to leave only a few micrometers between the sample and the detector, drastically decreasing particle energy loss (27) before reaching the detection region $(\sim 2 \mathrm{keV})$ compared with a conventional design built with a catheter (e.g., polyethylene tubing, Intramedic PE50 or PE10 [Becton Dickinson]) ( $\sim 200 \mathrm{keV})$.

The channel was microfabricated over a $44 \times 22 \mathrm{~mm}^{2}$ die containing a linear array of eight $2 \times 2 \mathrm{~mm}^{2}$ silicon $p-i-n$ photodiodes from Excelitas Technologies. The detailed fabrication process was reported elsewhere (27) and is briefly summarized in the supplemental materials. The resulting microfluidic chips had a $37 \times 1125 \mu \mathrm{m}^{2}$ cross section and 16-mm length for a detection volume of $0.66 \mu \mathrm{L}$.

As a first validation of the microfluidic blood-counting system, energy spectra were recorded at the shaping amplifier output and confirmed the validity of the measurement (25). Briefly, low-energy gammas and $\mathrm{x}$-rays from ${ }^{241} \mathrm{Am}$ and ${ }^{133} \mathrm{Ba}$ sources provided a calibration factor of $4.38 \mathrm{mV} / \mathrm{keV}$ and a noise level of $25 \mathrm{keV}$. Liquid sources showed a characteristic continuous spectrum for a $\beta$-emitter $\left({ }^{18} \mathrm{~F}\right)$ and a peak for a CE emitter $\left({ }^{99 \mathrm{~m}} \mathrm{Tc}\right)$.

\section{Catheter-Based Detector}

To characterize the gain in performance attributable to the microfluidics design, comparative measurements were conducted with a commercially packaged $p-i-n$ photodiode $\left(\mathrm{S} 3588-08,3 \times 30 \mathrm{~mm}^{2}\right.$ with $\sim 500 \mu \mathrm{m}$ epoxy protection layer, Hamamatsu Corp.) and a catheter bonded to its surface as pictured in Figure 2B.

\section{Data Corrections}

To be used as an AIF in pharmacokinetic models, the data recorded by the microfluidic blood-counting system must be corrected for radionuclide decay time, detector sensitivity, radioactivity dispersion, and propagation delay between the cannulation point and the detector. The system was also assessed for dead time, sensitivity to background radioactivity, and influence of sample temperature (supplemental materials). For all experiments, the detection threshold was adjusted to record a 5-cps background counting rate before injection of the radioactivity (8), and all recorded counting rates were decay-corrected by software referred to the acquisition start.

Sensitivity. The sensitivity relates the measured counting rate to the actual radioactive concentration in the detection volume. For direct $\beta$-detectors, the sensitivity is dependent on the incident particle energy 


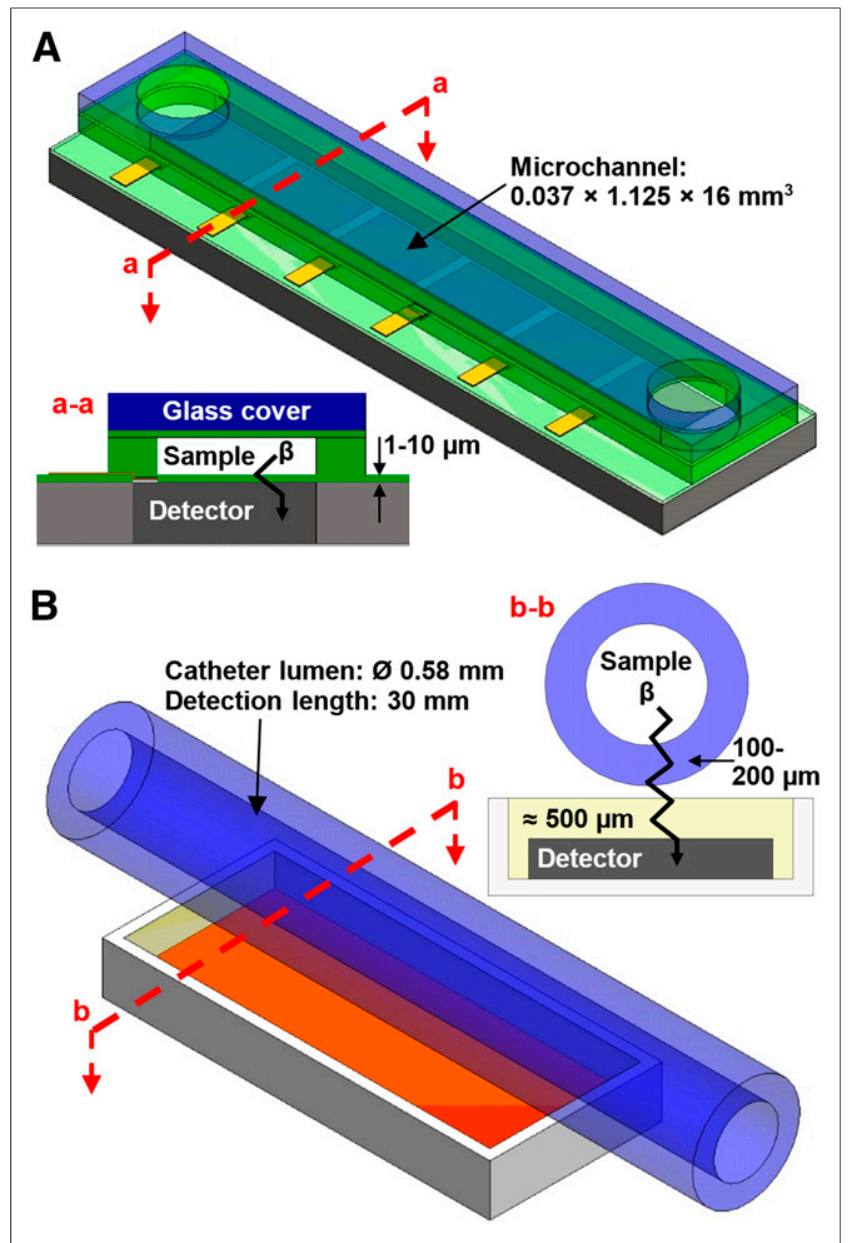

FIGURE 2. Three-dimensional and cross-sectional views of microfluidic chip (A) and of catheter-based geometry for $\beta$-detection (B) (not to scale).

and specific detection geometry; therefore, the sensitivity has to be characterized for each radionuclide. The microfluidic chip and a piece of PE50 or PE10 bonded to the catheter-based detector were consecutively injected with $10 \mathrm{kBq} / \mu \mathrm{L}$ solutions of four common PET radioisotopes $\left({ }^{18} \mathrm{~F},{ }^{11} \mathrm{C},{ }^{64} \mathrm{Cu}\right.$, and $\left.{ }^{68} \mathrm{Ga}\right)$ and a $40 \mathrm{kBq} / \mu \mathrm{L}$ solution of the SPECT isotope ${ }^{99 \mathrm{~m}} \mathrm{Tc}$. The detection threshold was adjusted, and data acquisition started for $2 \mathrm{~min}$. The average counting rate, $m$ (cps), corrected for radioactive decay, was used to calculate the device absolute detection efficiency $\eta(\%)$ for each radioisotope, following:

$$
\eta=\frac{m}{C \times V}
$$

where $C(\mathrm{~Bq} / \mu \mathrm{L})$ is the calibrated solution concentration, and $V(\mu \mathrm{L})$ is the device detection volume. Minimum and maximum detectable activities $(\mathrm{Bq} / \mu \mathrm{L})$ were then computed as:

$$
\begin{aligned}
& A_{\text {min }}=\frac{2.706+4.653 \times \sqrt{b}}{\eta \times V}, \\
& A_{\text {max }}=\frac{N_{\max }}{\eta \times V},
\end{aligned}
$$

where $b=5 \mathrm{cps}$ is the background counting rate, and $N_{\max }=65,535$ cps is the maximum counting rate allowed by the control unit 16-bit counter. Equation 2, derived from the frequently quoted Curie equation, can be interpreted as the minimum activity ensuring a false-positive readout less than $5 \%$ of the time (28).

Propagation Delay. The dead volume between the cannulation point and the detector causes a propagation delay. For typical mouse studies, the fluid path consists of a 20-cm-long PE10 tubing $(12 \mu \mathrm{L})$ from the animal to the detection unit entry and an 8-cm-long PE10 tubing $(5 \mu \mathrm{L})$ inside the detection unit. The fluidic junctions to tubing have no dead volume. Hence, the total dead volume is $17 \mu \mathrm{L}$ and the propagation delay is $35 \mathrm{~s}$ at $30 \mu \mathrm{L} / \mathrm{min}$ and $74 \mathrm{~s}$ at $14 \mu \mathrm{L} / \mathrm{min}$.

Dispersion. Dispersion effects produce distortion of transient radioactive concentrations in catheters, and may have a great impact on pharmacokinetic parameters, especially when estimating fast bloodtissue exchange parameters, such as in blood flow measurements $(29,30)$. Dispersion depends on several factors, including catheter internal diameter, withdrawal rate, and distance between the animal and detector. Fluidic junctions between the catheter and the microfluidic chip may also contribute to dispersion. The correction procedure for dispersion is based on a fit of the measured AIF using a biexponential model (the supplemental materials provide a detailed description).

To validate the method, an AIF measurement was simulated with radioactive water, corrected for dispersion and compared with manual samples. A reservoir was filled with $2 \mathrm{~mL}$ of cold water and placed on an agitating plate. The reservoir was sampled at 14 or $30 \mu \mathrm{L} / \mathrm{min}$ through a 20-cm-long PE10 catheter connected to the microfluidic counting system. ${ }^{18} \mathrm{~F}-\mathrm{FDG}$ was injected $30 \mathrm{~s}$ after starting the acquisition (21.6 MBq, $0.3 \mathrm{~mL}$ at $0.9 \mathrm{~mL} / \mathrm{min}$ using a syringe pump). A slow injection of cold water was started at the same time to simulate the tracer clearance ( $10 \mathrm{~mL}$ at $7 \mathrm{~mL} / \mathrm{min}$ using another syringe pump). Manual samples $(25 \mu \mathrm{L})$ were withdrawn using a micropipette every $10 \mathrm{~s}$ for $90 \mathrm{~s}$, then every $15 \mathrm{~s}$ for $30 \mathrm{~s}$ and every $2 \mathrm{~min}$ up to the end of the 10-min acquisition. Each sample was weighted to assess the exact volume. The samples' radioactivity was then measured using a $\gamma$-well counter (Cobra II Auto- $\gamma$; Canberra-Packard). AIF obtained both from manual samples and from the blood counter were corrected for radioactive decay and for the sensitivity of each counter. The latter was further corrected for propagation delay. A third AIF was obtained by correcting the AIF from the blood counter for dispersion as indicated above. To assess the efficiency of this correction, the three AIFs were compared using a repeated-measures 1-way ANOVA test with the Greenhouse-Geisser correction and Dunnett multiple comparison using the manual samples as a reference. The curves were considered significantly different for a $P$ value of 0.05 or less.

\section{Animal Studies}

Animal Preparation. Animal experiments were performed in accordance with the recommendations of the in-house Ethics Committee for Animal Experiments and the Canadian Council on Animal Care. BALB/c and C57BL/6 mice had free access to food and water before the experiment. The animals were anesthetized with isoflurane $(2 \%+$ $1.5 \mathrm{~L} / \mathrm{min} \mathrm{O}_{2}$ ) and cannulated in the caudal vein for injection and in the carotid artery for blood withdrawal (20-cm-long PE10 catheter prefilled with heparinized saline, $0.9 \%, 50 \mathrm{U} / \mathrm{mL}$ ). The animal temperature was regulated, and the heart beat and breathing were monitored to ensure that physiologic conditions were maintained stable during the experiment.

PET Pharmacokinetic Study. A study was performed to investigate the measurement of myocardial blood flow and oxygen consumption in a mouse with ${ }^{11} \mathrm{C}$-acetate using the microfluidic blood-counting system. This study yielded an AIF from PET images in addition to the AIF measured by the blood counter. A cannulated BALB/c mouse (28 g) was placed in a LabPET4 scanner (31) (Gamma Medica Inc.) with a transaxial and axial field of view of 11 and $3.75 \mathrm{~cm}$, respectively, 
TABLE 1

Absolute Detection Efficiency and Detection Limit for Microfluidic and Catheter-Based Detectors

\begin{tabular}{|c|c|c|c|c|c|c|c|c|c|c|}
\hline \multirow[b]{2}{*}{ Isotope } & \multirow[b]{2}{*}{$\begin{array}{c}E_{\beta m e a n} \\
(\mathrm{keV})\end{array}$} & \multirow[b]{2}{*}{$\begin{array}{l}\beta / C E \text { emission } \\
\text { probability (\%) }\end{array}$} & \multicolumn{2}{|c|}{$\begin{array}{c}\text { Chip } 1 \\
(0.66 \mu \mathrm{L})\end{array}$} & \multicolumn{2}{|c|}{$\begin{array}{l}\text { Chip 2* } \\
(1.46 \mu \mathrm{L})\end{array}$} & \multicolumn{2}{|c|}{$\begin{array}{c}\text { PE10 } \\
(1.85 \mu \mathrm{L})\end{array}$} & \multicolumn{2}{|c|}{$\begin{array}{c}\text { PE50 } \\
(7.93 \mu \mathrm{L})\end{array}$} \\
\hline & & & $\begin{array}{c}\eta \\
\text { (cps/Bq) }\end{array}$ & $\begin{array}{c}A_{\min }-A_{\max } \\
(\mathrm{Bq} / \mu \mathrm{L})\end{array}$ & $\begin{array}{c}\eta \\
(\mathrm{cps} / \mathrm{Bq})\end{array}$ & $\begin{array}{c}A_{\min }-A_{\max } \\
(\mathrm{Bq} / \mu \mathrm{L})\end{array}$ & $\begin{array}{c}\eta \\
\text { (cps/Bq) }\end{array}$ & $\begin{array}{c}A_{\min }-A_{\max } \\
(\mathrm{Bq} / \mu \mathrm{L})\end{array}$ & $\begin{array}{c}\eta \\
(\mathrm{cps} / \mathrm{Bq})\end{array}$ & $\begin{array}{c}A_{\min }-A_{\max } \\
(\mathrm{Bq} / \mu \mathrm{L})\end{array}$ \\
\hline${ }^{64} \mathrm{Cu}$ & 190,278 & $37.2,17.8$ & $20 \%$ & $100-5.0 \cdot 10^{5}$ & $20 \%$ & $45-2.3 \cdot 10^{5}$ & $3 \%$ & $254-1.3 \cdot 10^{6}$ & $2 \%$ & $93-4.6 \cdot 10^{5}$ \\
\hline${ }^{18} \mathrm{~F}$ & 250 & 96.7 & $39 \%$ & $51-2.5 \cdot 10^{5}$ & $39 \%$ & $23-1.2 \cdot 10^{5}$ & $7 \%$ & $103-5.1 \cdot 10^{5}$ & $4 \%$ & $38-1.9 \cdot 10^{5}$ \\
\hline${ }^{11} \mathrm{C}$ & 386 & 99.8 & $47 \%$ & $42-2.1 \cdot 10^{5}$ & $47 \%$ & $19-9.6 \cdot 10^{4}$ & $15 \%$ & $46-2.3 \cdot 10^{5}$ & $12 \%$ & $14-6.9 \cdot 10^{4}$ \\
\hline${ }^{68} \mathrm{Ga}$ & 353,836 & $1.2,87.7$ & $43 \%$ & $47-2.3 \cdot 10^{5}$ & $43 \%$ & $21-1.1 \cdot 10^{5}$ & $22 \%$ & $33-1.6 \cdot 10^{5}$ & $17 \%$ & $10-4.9 \cdot 10^{4}$ \\
\hline${ }^{99 m T c}$ & 120,138 & $8.8,1$ & $4 \%$ & $527-2.6 \cdot 10^{6}$ & $4 \%$ & $238-1.2 \cdot 10^{6}$ & $0.2 \%$ & $4,447-2.2 \cdot 10^{7}$ & $0.2 \%$ & $1,034-5.2 \cdot 10^{6}$ \\
\hline
\end{tabular}

${ }^{*}$ Estimated.

$E_{\beta \text { mean }}=$ mean energy of $\beta$-particles.

Detection volume is indicated in brackets for each device. Data for microfluidic Chip 2 were computed.

and a nearly isotropic resolution of $1.35 \mathrm{~mm}$. The heart was centered in the field of view, and the withdrawing cannula was connected to the microfluidic blood-counting system. To minimize the cannula length and keep it straight, the blood counter was placed at the rear of the scanner. The discriminator threshold of the blood counter was then adjusted, and the pump speed was set to $14 \mu \mathrm{L} / \mathrm{min}$. Both the PET and the counter acquisitions were started in synchronization $30 \mathrm{~s}$ before the injection of $40.2 \mathrm{MBq}$ of ${ }^{11} \mathrm{C}$-acetate $(0.1 \mathrm{~mL}$ at $0.2 \mathrm{~mL} / \mathrm{min})$ in the animal. The blood drawing rate was lowered to $10 \mu \mathrm{L} / \mathrm{min}$ after $4 \mathrm{~min}$ and kept unchanged up to the end of the 20.5-min acquisition. After the experiment, a cylindric mouse phantom $(1.2 \mathrm{kBq} / \mu \mathrm{L}, 26.6 \mathrm{~mL})$ was scanned to calibrate the PET images.

Technetium AIF Measurement. A second mouse study was performed to validate the AIF measurement using the SPECT radiotracer ${ }^{99} \mathrm{mcO}_{4}{ }^{-}$. A C57BL/6 mouse (28 g) was anesthetized and cannulated as described above. A 45-min acquisition was then started on the microfluidic blood-counting system $(14 \mu \mathrm{L} / \mathrm{min})$, and $88 \mathrm{MBq}$ $(0.15 \mathrm{~mL}$ at $0.3 \mathrm{~mL} / \mathrm{min})$ of ${ }^{99} \mathrm{mcO}_{4}{ }^{-}$were injected $1 \mathrm{~min}$ later. After $5 \mathrm{~min}$, the drawing rate was lowered to $10 \mu \mathrm{L} / \mathrm{min}$ for the remaining time. For this experiment, the input of the blood counter was switched back to nonradioactive saline after 43 min.

Data Analysis and Corrections. AIFs measured with the blood counter were corrected for decay time, sensitivity, propagation delay, and dispersion. The measured data, recorded every second, were also smoothed using a 10-s moving average filter to reduce statistical noise caused by the random nature of radioactive decay. PET images were reconstructed using a maximum-likelihood estimator algorithm with 15 iterations and using the dynamic sequence $1 \times 30$ (before injection), $12 \times 5,8 \times 30,2 \times 150$, and $2 \times 300 \mathrm{~s}$ ( 25 frames). Attenuation and scatter corrections were not applied. Myocardium time-activity curves (mTAC) and image-derived input functions (IDIF) were obtained by manually drawing regions-of-interest (ROIs) on the myocardium and left ventricle, respectively, in the summed frames of the interval from 2 to $3 \mathrm{~min}$. IDIF was corrected for spill-in effects assuming an ROI volume of $2.5 \mathrm{~mm}^{3}$, and both mTAC and IDIF were corrected for partial volume considering an ROI average diameter of $2.5 \mathrm{~mm}$ (32). A 3-compartment kinetic model was used for computing myocardial blood flow $\left(K_{1}\right)$ and oxygen consumption $\left(k_{2}\right)$ using the equations described in the supplemental materials. For validation, we performed a comparison between $K_{1}$ and $k_{2}$ parameters estimated using IDIF or AIF obtained from the microfluidic blood-counting system.

\section{RESULTS}

\section{Data Corrections}

Sensitivity. Sensitivity parameters computed using Equations 1-3 are summarized in Table 1 for different isotopes measured by microfluidic and catheter-based detectors. Absolute detection sensitivities $(\eta)$ of the microfluidic detector for PET isotopes ranged from $20 \%$ to $47 \%$, a 2 - to 10 -fold gain relative to the conventional catheter-based configurations. Minimum detectable activities $\left(A_{\min }\right)$ of PET isotopes ranged from 42 to $100 \mathrm{~Bq} / \mu \mathrm{L}$ for Chip 1 , whereas they ranged from 33 to $254 \mathrm{~Bq} / \mu \mathrm{L}$ for PE10 and from 10 to $93 \mathrm{~Bq} / \mu \mathrm{L}$ for PE50. These sensitivities are suitable for most pharmacokinetic PET studies in small animals. PE50 yields lower $A_{\text {min }}$ but has a larger detection volume requiring more blood. For comparison purposes, estimates for Chip 2 with a larger detection volume $\left(37 \times 1,250 \mu \mathrm{m}^{2}\right.$ cross section, 31.5-mm detection length) were added in Table 1, assuming the same $\eta$ as Chip 1. The resulting detection volume was $1.46 \mu \mathrm{L}$, yielding $A_{\text {min }}$ ranging from 19 to $45 \mathrm{~Bq} / \mu \mathrm{L}$, up to twice lower than that obtained with PE50 having a detection volume of $7.93 \mu \mathrm{L}$. Finally, the microfluidic blood-counting system was able to record an AIF with ${ }^{99 \mathrm{~m}} \mathrm{Tc}\left(A_{\min }=238-527 \mathrm{~Bq} / \mu \mathrm{L}\right)$, which was not possible with the conventional catheter-based geometry $\left(A_{\min }>1 \mathrm{kBq} / \mu \mathrm{L}\right)$. The maximum detectable activity ranged from 50 to $1,267 \mathrm{kBq} / \mu \mathrm{L}$ for PET isotopes and was above $500 \mathrm{kBq} / \mu \mathrm{L}$ for ${ }^{99 \mathrm{~m}} \mathrm{Tc}$.

Dispersion. The AIF measured from manual samples and from the microfluidic blood-counting system with and without the correction for dispersion are shown in Figure 3. The ANOVA test showed that the blood counter curve corrected for dispersion was not statistically different from manual samples $(P=0.03)$, whereas it was without the correction $(P=0.74)$. The resulting fitted dispersion time constant $\tau_{\text {disp }}$ was 5.4 and $2.8 \mathrm{~s}$ at 14 and $30 \mu \mathrm{L} / \mathrm{min}$ withdrawal rate, respectively.

\section{Animal Studies}

PET Pharmacokinetic Study. Typical IDIF and mTAC obtained from PET images and the corrected AIF obtained from the microfluidic blood-counting system are illustrated in Figures 4A and $4 \mathrm{~B}$, respectively, together with the PET images of the mouse heart for the 2- to 3-min time frame and the ROIs used to compute the mTAC and IDIF (Fig. 4C). The resulting myocardial blood flow and oxygen consumption indices given by the rate constants 


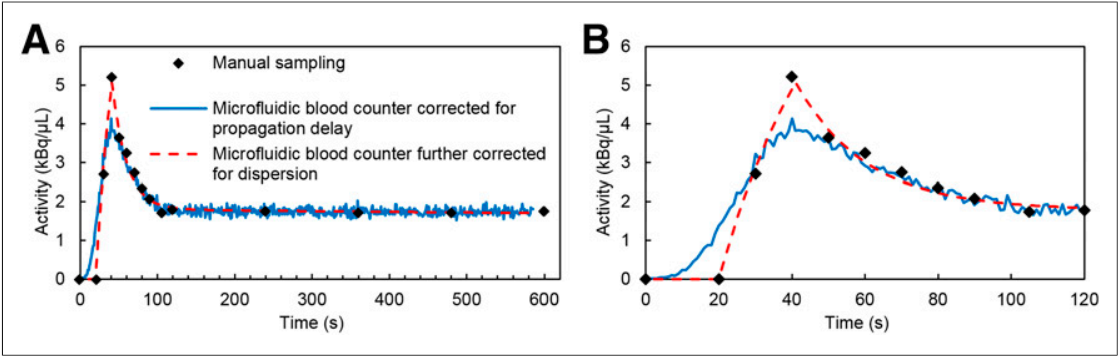

FIGURE 3. (A) AIF simulated by injecting ${ }^{18} \mathrm{~F}-\mathrm{FDG}(21.6 \mathrm{MBq}, 0.3 \mathrm{~mL}$ at $0.9 \mathrm{~mL} / \mathrm{min})$ in reservoir while flushing with cold water ( $10 \mathrm{~mL}$ at $7 \mathrm{~mL} / \mathrm{min})$. AlFs were obtained manually (micropipette) and with microfluidic blood-counting system (14 $\mu \mathrm{L} / \mathrm{min}$ withdrawal) to validate dispersion correction. (B) Detailed view of bolus region.

$K_{1}$ and $k_{2}$ are 3.4 and $0.9 \mathrm{~min}^{-1}$, respectively, using the IDIF as the blood curve in the kinetic model. These values are 3.5 and $0.7 \mathrm{~min}^{-1}$ using the AIF from the microfluidic blood-counting system corrected for dispersion. These values are in good agreement with values of $K_{1}=3.5 \pm 1.0 \mathrm{~min}^{-1}$ and $k_{2}=1.3 \pm$ $0.5 \mathrm{~min}^{-1}$ obtained in rats with a similar protocol (32). The dispersion time constant fitted by the dispersion correction model was $\tau_{\text {disp }}=9.4 \mathrm{~s}$. As expected from fluid viscosities, the dispersion is higher in this experiment performed with blood than in the previous one performed with water for an identical setup.

Technetium AIF Measurement. An AIF obtained with the microfluidic blood counter after ${ }^{99 \mathrm{~m}} \mathrm{Tc}$ injection in a mouse is shown in Figure 5 with and without correction for dispersion. This experiment confirms the ability of the microfluidic system to measure AIF with the SPECT isotope ${ }^{99 \mathrm{~m}} \mathrm{Tc}$ emitting only low-energy conversion electrons. It also confirmed the system's ability to perform data acquisition up to $40 \mathrm{~min}$ without blood clotting and without any significant tracer adsorption to the microfluidic chip walls, because the counting rate returned to background level when nonradioactive saline was injected in the microfluidic counter.

\section{DISCUSSION}

\section{Microfluidic Blood-Counting System}

This work describes a microfluidic blood-counting system to monitor blood radioactivity from PET and SPECT radiotracers with high efficiency and high temporal resolution in a small blood

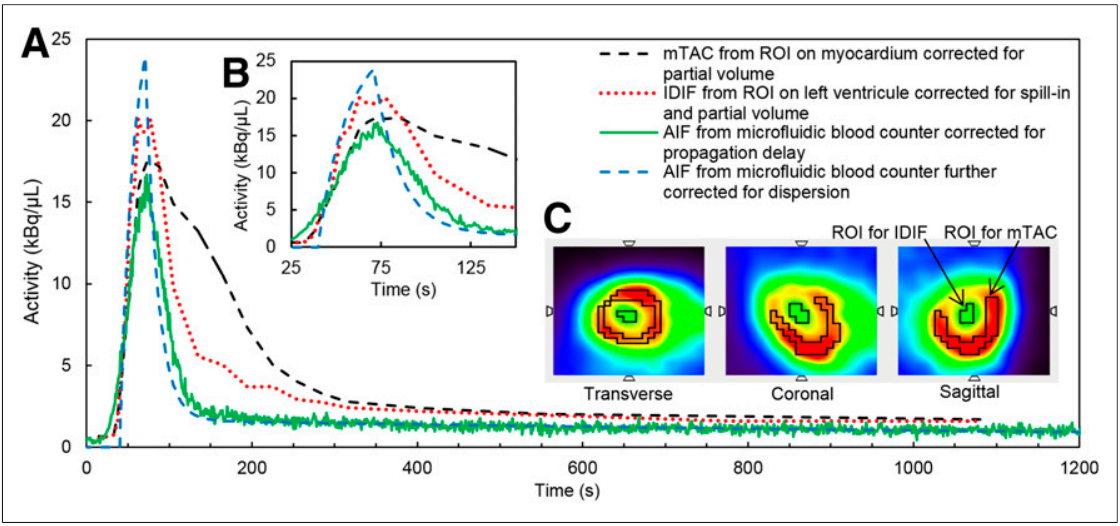

FIGURE 4. (A) AIF, IDIF, and mTAC obtained after bolus injection of ${ }^{11} \mathrm{C}$-acetate $(40.2 \mathrm{MBq}$ in $0.1 \mathrm{~mL}$ at $0.2 \mathrm{~mL} / \mathrm{min}$, no flush) in $28-\mathrm{g}$ BALB/c mouse. AlF was obtained at $14 \mu \mathrm{L} / \mathrm{min}$ withdrawa rate for $4 \mathrm{~min}$, then at $10 \mu \mathrm{L} / \mathrm{min}$ for remainder of experiment. (B) Detailed view of bolus region. (C) IDIF and mTAC were calculated from ROls on left ventricle and myocardium, respectively. volume. The technology is compatible with integration of other on-chip functionalities such as plasma separation from wholeblood and plasma sampling and analysis as a function of time to increase the throughput of new radiotracers development.

The invasiveness of external AIF measurement is often mentioned as a drawback that makes follow-up studies difficult, especially when the animal is cannulated in the carotid artery as in the studies presented in this work. Blood withdrawal is, however, necessary when chemical analysis of plasma samples is required to quantify metabolites of new radiotracers. Less invasive cannulation points such as the tail artery can then be adopted in conjunction with a microfluidic blood-counting system (33).

Sensitivity. The proposed microfluidic blood-counting system shows high absolute detection efficiency for PET tracers, especially for isotopes with the highest $E_{\beta \max }$, that is, ${ }^{11} \mathrm{C}$ and ${ }^{68} \mathrm{Ga}$. The achieved efficiencies, respectively, $47 \%$ and $43 \%$, are almost ideal considering that the emission is random and that the detector covers only 1 side of the microchannel.

The $\beta$-emitting isotopes with lower energy, ${ }^{18} \mathrm{~F}$ and ${ }^{64} \mathrm{Cu}$, have slightly reduced detection efficiency because a larger fraction of the particles do not reach the detector or produce a signal below the noise threshold. The detection efficiency of ${ }^{64} \mathrm{Cu}$ is also affected by a $\beta$-emission probability of only $56 \%$. Thanks to both, the thin interface thickness between the sample and detector and the more efficient detection geometry, the loss of detection efficiency for low-energy isotopes is much less significant with the microfluidic system, improving from more than a 5-fold drop between ${ }^{11} \mathrm{C}$ and ${ }^{64} \mathrm{Cu}$ for catheter-based detectors to only slightly more than a 2 -fold reduction for the microfluidic detector.

The gain in sensitivity achieved with the current microfluidic device would still not be sufficient to be applicable for use in human studies, considering typical peak and equilibrium blood tracer concentrations of 74 and $\sim 7 \mathrm{~Bq} / \mu \mathrm{L}$, respectively. However, microfluidic devices with larger detection volumes (Table 1, Chip 2) and optimized detection geometry can readily be designed to achieve the required sensitivity.

Dispersion Correction. Kinetic parameters are estimated using the AIF and timeactivity curve as inputs. However, dispersion of the radioactive bolus inside the catheter of an external blood counter distorts the AIF relative to the sampling site and leads to error in kinetic parameters. For example, Votaw et al. (34) measured a $0.3 \%$ error in cerebral blood flow estimated with $\mathrm{H}_{2}{ }^{15} \mathrm{O}$ for a dispersion of $1.3 \mathrm{~s}$. This error rises to $33 \%$ for a dispersion of $10 \mathrm{~s}$. Dispersion can be reduced by minimizing the animalto-detector distance, by decreasing the cannula internal diameter, and by maximizing the withdrawal rate. Unfortunately, dispersion is always significant in typical experiments with mice, mainly because of the limited withdrawal rate. If the injection site is far from the sampling site, dispersion 


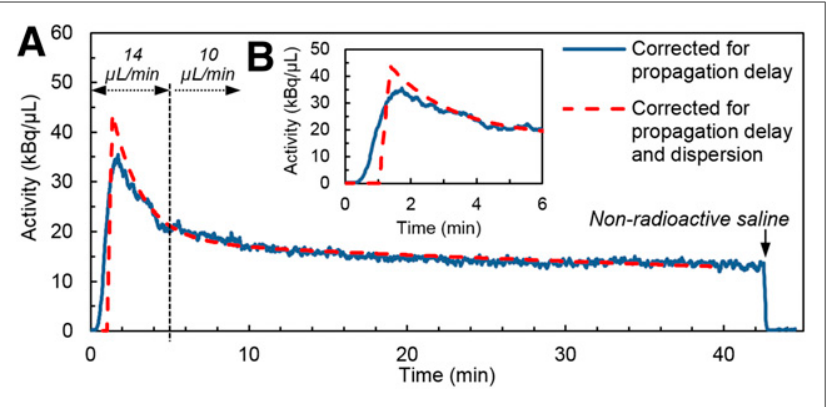

FIGURE 5. AIF measured with microfluidic blood counter after bolus

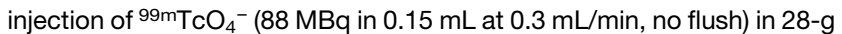
C57BL/6 mouse. Withdrawal rate was reduced after $5 \mathrm{~min}$. Measured data were corrected for radioactive decay and smoothed to decrease statistical error (moving average, $10 \mathrm{~s}$ ).

inside the animal can also affect the measured AIF. Such dispersion should, however, be considered by the biexponential model used for correction (35).

The method implemented here to correct for dispersion yields satisfactory results. Compared with other methods reported in the literature, fitting of the measured AIF has the advantage of requiring no previous parameter estimation. However, initial parameters, especially the injection time, must have been well recorded to obtain reproducible results. In the present study (supplemental materials), two consecutive fits were made, the first one to have a good estimation of input parameters and the second for more accurate fitting of the tracer in vivo kinetics.

In one validation study, the experimentally simulated AIF was compared with manual sampling. Even if manual sampling is considered as the gold standard for AIF measurements, its temporal resolution is limited and may induce distortion in the AIF too (33). Another common reference for nondispersed AIF is the IDIF extracted from a ROI in PET images. However, the temporal resolution in dynamic image series can also be limited and such measurements require further partial-volume and spill-in corrections that may also introduce additional errors.

\section{Animal Studies}

The proposed microfluidic blood-counting system was successfully used with rats (27) and mice in this work after injection of PET and SPECT tracers. The counter-derived AIF was successfully compared with manual samples in a phantom experiment. Direct comparison of the AIF to IDIF in the present PET study with mice was not straightforward: on one hand, AIF must be corrected for propagation delay and dispersion; and on the other hand, IDIF is affected by partial volume and myocardium activity spilling in the left ventricle. Although the rising edges were similar, some contamination was visible on the falling edge of the IDIF (Fig. 4), even when applying spill-in correction. Moreover, the sampling period for IDIF is limited to approximately $5 \mathrm{~s}$ in practice, which induces undesirable smoothing of the peak corresponding to the bolus injection and reduces its maximum. All these factors influence the reliability and accuracy of pharmacokinetic parameters calculated from $\operatorname{IDIF}(4,5)$. Also, only wholeblood radioactivity can be estimated from IDIF, limiting its use to known radiotracers. In all those respects, the microfluidic bloodcounting system will be an attractive alternative to IDIF and manual sampling for AIF measurements.

\section{CONCLUSION}

A blood-counting system, based on the detection of $\beta$-particles in a microfluidic chip, was developed to monitor the radioactivity in rodents' blood in real time with high efficiency using a small blood volume. The system comprises dedicated electronics, software, and pumping unit for stand-alone use in PET and SPECT studies. Sensitive measurements can be performed with high temporal resolution for pharmacokinetic studies in mice. Furthermore, the microfluidic design enables the detection of ${ }^{99 m}$ Tc-based SPECT tracers, which is not possible with catheterbased $\beta$-detection systems. Experiments showed that the system was insensitive to background radioactivity and blood temperature and that no dead time correction was required. Simple and robust corrections were successfully applied for delay and dispersion. Microfluidics provides a reliable and attractive solution for blood radioactivity measurement in pharmacokinetic studies with small animals.

\section{DISCLOSURE}

The costs of publication of this article were defrayed in part by the payment of page charges. Therefore, and solely to indicate this fact, this article is hereby marked "advertisement" in accordance with 18 USC section 1734. This work was supported by the Canadian Institutes of Health Research (CIHR) grant \#MOP86717 and NanoQuébec. The Research Centre of CHUS (CRCHUS) hosting the Sherbrooke Molecular Imaging Centre is a FRQS-funded research organization. No other potential conflict of interest relevant to this article was reported.

\section{ACKNOWLEDGMENTS}

We thank Dr. Henri Dautet of Excelitas Technologies for supplying the $p-i-n$ photodiode wafers, Véronique DumulonPerreault and Mélanie Archambault from the Sherbrooke Molecular Imaging Centre for support with animal experiments, Sylvain Lauzier and Jean-Nicolas Mélançon-Emond from the Department of Electrical and Computer Engineering for help with the electronic boards, and the staff of the Nanofabrication and Nanocharacterisation Research Center (CRN2) of Université de Sherbrooke for microfabrication support.

\section{REFERENCES}

1. Bonekamp D, Hammoud DA, Pomper MG. Molecular imaging: techniques and current clinical applications. Appl Radiol. 2010;39:10-21.

2. Kagadis GC, Loudos G, Katsanos K, Langer SG, Nikiforidis GC. In vivo small animal imaging: current status and future prospects. Med Phys. 2010;37:6421-6442.

3. Dupont P, Warwick J. Kinetic modelling in small animal imaging with PET. Methods. 2009;48:98-103.

4. Croteau É, Lavallée É, Labbe SM, et al. Image-derived input function in dynamic human PET/CT: methodology and validation with ${ }^{11} \mathrm{C}$-acetate and ${ }^{18} \mathrm{~F}$-fluorothioheptadecanoic acid in muscle and ${ }^{18} \mathrm{~F}$-fluorodeoxyglucose in brain. Eur J Nucl Med Mol Imaging. 2010;37:1539-1550.

5. Mabrouk R, Dubeau F, Bentabet L. Dynamic cardiac PET imaging: extraction of time-activity curves using ICA and a generalized Gaussian distribution model. IEEE Trans Biomed Eng. 2013;60:63-71.

6. Warnock G, Bahri M-A, Giacomelli F, et al. Use of a beta microprobe system to measure arterial input function in PET via an arteriovenous shunt in rats. EJNMMI Res. 2011;1:13.

7. Seki C, Okada H, Mori S, et al. Application of a beta microprobe for quantification of regional cerebral blood flow with ${ }^{15} \mathrm{O}$-water and PET in rhesus monkeys. Ann Nucl Med. 1998;12:7-14.

8. Convert L, Morin-Brassard G, Cadorette J, Archambault M, Bentourkia M, Lecomte R. A new tool for molecular imaging: the microvolumetric beta blood counter. J Nucl Med. 2007;48:1197-1206. 
9. Roehrbacher F, Bankstahl JP, Bankstahl M, et al. Development and performance test of an online blood sampling system for determination of the arterial input function in rats. EJNMMI Phys. 2015;2:1.

10. Yamamoto S, Imaizumi M, Shimosegawa E, et al. A compact and high sensitivity positron detector using dual-layer thin GSO scintillators for a small animal PET blood sampling system. Phys Med Biol. 2010;55:3813-3826.

11. Whitesides GM. The origins and the future of microfluidics. Nature. 2006;442:368-373.

12. Haeberle S, Zengerle R. Microfluidic platforms for lab-on-a-chip applications. Lab Chip. 2007;7:1094-1110.

13. Henderson E, Rutt BK, Lee T-Y. Temporal sampling requirements for the tracer kinetics modeling of breast disease. Magn Reson Imaging. 1998;16:1057-1073.

14. Wu H-M, Sui G, Lee C-C, et al. In vivo quantitation of glucose metabolism in mice using small-animal PET and a microfluidic device. J Nucl Med. 2007;48:837-845.

15. Kimura Y, Seki C, Hashizume N, et al. Novel system using microliter order sample volume for measuring arterial radioactivity concentrations in whole blood and plasma for mouse PET dynamic study. Phys Med Biol. 2013;58:7889-7903.

16. Arlinghaus HF, Kwoka MN, Jacobson KB. Analysis of biosensor chips for identification of nucleic acids. Anal Chem. 1997;69:3747-3753.

17. Lavén M, Wallenborg S, Velikyan I, et al. Radionuclide imaging of miniaturized chemical analysis systems. Anal Chem. 2004;76:7102-7108.

18. Tseng W-Y, Cho JS, Ma X, Kunihiro A, Chatziioannou AF, Van Dam RM. Toward reliable synthesis of radiotracers for positron emission tomography in PDMS microfluidic chips: study and optimization of the $\left[{ }^{18} \mathrm{~F}\right]$ fluoride drying process. NSTI-Nanotech 2010. 2010;2:472-475.

19. Vaccari L, Birada G, Grenci G, Pacor S, Businaro L. Synchrotron radiation infrared microspectroscopy of single living cells in microfluidic devices: advantages, disadvantages and future perspectives. J Phys Conf Ser. 2012;359:012007.

20. Dhouib K, Malek CK, Pfleging W, et al. Microfluidic chips for the crystallization of biomacromolecules by counter-diffusion and on-chip crystal X-ray analysis. Lab Chip. 2009;9:1412-1421.

21. Burns MA, Mastrangelo CH, Sammarco TS, et al. Microfabricated structures for integrated DNA analysis. Proc Natl Acad Sci USA. 1996;93:5556-5561.

22. Burns MA, Johnson BN, Brahmasandra SN, et al. An integrated nanoliter DNA analysis device. Science. 1998;282:484-487.

23. Dooraghi AA, Vu NT, Silverman RW, et al. Betabox: a beta particle imaging system based on a position sensitive avalanche photodiode. Phys Med Biol. 2013;58:3739-3753.
24. Convert L, Girard Baril F, Boisselle V, et al. Microfluidic beta and conversion electron radiation detector for preclinical pharmacokinetic studies with PET and SPECT radiotracers. IEEE Nucl Sci Symp Conf Rec. 2010:2128-2131.

25. Convert L, Girard-Baril F, Renaudin A, et al. High efficiency microfluidic beta detector for pharmacokinetic studies in small animals. Nucl Instrum Methods Phys Res A. 2011;652:735-738.

26. Convert L, Chabot V, Zermatten P-J, et al. Passivation of KMPR microfluidic channels with bovine serum albumin (BSA) for improved hemocompatibility characterized with metal-clad waveguides. Sens Actuators B Chem. 2012;173:447454.

27. Convert L, Girard-baril F, Boisselle V, et al. Blood compatible microfluidic system for pharmacokinetic studies in small animals. Lab Chip. 2012;12:46834692 .

28. Knoll GF. Radiation Detection and Measurement. 3rd ed. New York, NY: John Wiley and Sons, 2000:96.

29. Kanno I, Iida H, Miura S, et al. A system for cerebral blood flow measurement using an $\mathrm{H}_{2}{ }^{15} \mathrm{O}$ autoradiographic method and positron emission tomography. J Cereb Blood Flow Metab. 1987;7:143-153.

30. Munk OL, Keiding S, Bass L. A method to estimate dispersion in sampling catheters and to calculate dispersion-free blood time-activity curves. Med Phys. 2008;35:3471-3481.

31. Bergeron M, Cadorette J, Beaudoin J-F, et al. Performance evaluation of the LabPET APD-based digital PET scanner. IEEE Trans Nucl Sci. 2009;56:10-16.

32. Croteau É, Gascon S, Bentourkia M, et al. $\left[{ }^{11} \mathrm{C}\right]$ acetate rest-stress protocol to assess myocardial perfusion and oxygen consumption reserve in a model of congestive heart failure in rats. Nucl Med Biol. 2012;39:287-294.

33. Croteau É, Poulin E, Tremblay S, et al. Arterial input function sampling without surgery in rats for positron emission tomography molecular imaging. Nucl Med Commun. 2014;35:666-676.

34. Votaw JR, Shulman SD. Performance evaluation of the pico-count flow-through detector for use in cerebral blood flow PET studies. J Nucl Med. 1998;39:509515.

35. Iida H, Kanno I, Miura S, Murakami M, Takahashi K, Uemura K. Error analysis of a quantitative cerebral blood flow measurement using $\mathrm{H}_{2}{ }^{15} \mathrm{O}$ autoradiography and positron emission tomography, with respect to the dispersion of the input function. J Cereb Blood Flow Metab. 1986;6:536-545 